\title{
Efecto de las Condiciones de Secado en Lecho Fluidizado Pulsante sobre el Contenido de Sulforafano de Brócoli
}

\author{
Andrea V. Mahnn ${ }^{(1) \star}$, Carmen E. Pérez ${ }^{(2)}$ y Alejandro E. Reyes ${ }^{(1)}$ \\ (1) Departamento de Ingeniería Química, Universidad de Santiago de Chile, Avenida Libertador Bernardo \\ O’Higgins 3363, Estación Central, Santiago, Chile. (e-mail: andrea.mahn@usach.cl; \\ alejandro.reyes@usach.cl) \\ (2) Departamento de ingeniería Agroindustrial, Universidad Pontificia Bolivariana, Cra. 6 No. 97A-99, \\ Montería, Colombia. (carmenelena.perez@upb.edu.co) \\ * Autor a quien debe ser dirigida la correspondencia
}

Recibido Ene. 20, 2017; Aceptado Mar. 15, 2017; Versión final May. 4, 2017, Publicado Ago. 2017

\begin{abstract}
Resumen
Se investigó el efecto de las condiciones de secado de brócoli enriquecido en sulforafano, en un secador de lecho fluidizado pulsante, sobre el contenido de sulforafano y la cinética de secado, para identificar las condiciones de operación que minimizan las pérdidas de sulforafano en el producto. Se utilizó un diseño central compuesto, cuyos factores fueron temperatura del aire de secado y velocidad de giro de la placa rotatoria. Las curvas de secado se ajustaron a modelos empíricos, encontrándose el mejor ajuste con el modelo de Page $\left(R^{2} \geq 99 \%\right)$. El tiempo de secado disminuyó al aumentar la temperatura del aire de secado y al aumentar la velocidad de giro de la placa giratoria. Las condiciones óptimas de secado predichas por el modelo de regresión fueron $29^{\circ} \mathrm{C}$ y $108 \mathrm{rpm}$, resultando en $1.5 \mu \mathrm{mol}$ sulforafano $/ \mathrm{g}$ (base seca), valor que representa una concentración de sulforafano 1.36 veces la del brócoli fresco. Este valor se confirmó experimentalmente.
\end{abstract}

Palabras clave: secador de lecho fluidizado pulsante; brócoli; sulforafano; cinética de secado; optimización

\section{Effect of the Drying Conditions in a Pulsed Fluidized Bed Dryer on the Sulforaphane Content of Broccoli}

\begin{abstract}
The effect of the drying conditions of sulforaphane-rich broccoli in a pulsed fluidized bed dryer, on the sulforaphane content and drying kinetics was studied, with the aim of identifying the operating conditions that minimize the loss of sulforaphane in the dehydrated product. A central composite design was used, in which drying air temperature and plate rotational speed were the variables considered. The drying curves were fitted to empirical models and it was found that Page's model showed the best fit $\left(R^{2} \geq 99 \%\right)$. The drying time decreased with the increase of drying air temperature and the increase of the plate rotational speed. The regression model predicted that $29^{\circ} \mathrm{C}$ and $108 \mathrm{rpm}$ were the optimal conditions, resulting in a sulforaphane content equal to $1.5 \mu \mathrm{mol} / \mathrm{g}$ in dry basis, giving a value that is 1.36 -fold the sulforaphane content in fresh broccoli. This value was experimentally confirmed.
\end{abstract}




\section{INTRODUCCIÓN}

El sulforafano es un isotiocianato que proviene de las plantas crucíferas, y es reconocido como el agente anticancerígeno de origen natural más poderoso que se conoce hasta ahora (Matusheski et al., 2004). Su precursor, la glucorafanina, se encuentra principalmente en brócoli, y por lo tanto esta verdura ofrece un gran potencial como fuente de sulforafano. Según datos de la FAO, la producción anual de brócoli en Sud América es de 72 mil toneladas, lo que corresponde al 1\% de la producción mundial. Se ha investigado el procesamiento de brócoli para aumentar el contenido de sulforafano (Wang et al., 2012; Matusheski et al., 2004). Pérez et al. (2014) informaron las condiciones de escaldado que maximizan el contenido de este compuesto en brócoli. Recientemente, Mahn y Pérez (2016) reportaron un proceso de incubación de brócoli escaldado, que permite obtener un $94 \%$ de conversión de glucorafanina en sulforafano. Una vez maximizado el contenido de sulforafano, se requiere de un proceso que permita la preservación del alimento y le confiera características físicas acorde a sus posibles aplicaciones industriales, tales como ingrediente funcional o nutracéutico. Al tratarse de un alimento enriquecido de manera natural en sulforafano, no se contravienen las normativas relativas a seguridad alimentaria.

Entre los métodos de preservación, el secado resulta ser uno de los más atractivos, debido a su versatilidad, costo relativamente bajo, y a las características físicas del producto obtenido. Una de las principales dificultades relativas al secado de brócoli rico en sulforafano es la alta termo labilidad del compuesto. Tanongkankit et al. (2011) y Lekcharoenkul et al. (2014) investigaron el secado convectivo con aire caliente de hojas de repollo fresco, informando grandes pérdidas de sulforafano atribuidas al tiempo de secado prolongado y a la alta temperatura del aire de secado $\left(70-80^{\circ} \mathrm{C}\right)$. Mahn et al. (2016a) reportaron la evolución del contenido de sulforafano en brócoli durante el secado convectivo con aire caliente, concluyendo que la degradación térmica del sulforafano se produce cuando la temperatura del brócoli supera los $40^{\circ} \mathrm{C}$. Por lo tanto, un proceso de secado a una temperatura tal que el brócoli no supere los $40^{\circ} \mathrm{C}$ debería mantener el contenido de sulforafano en el producto final.

Si bien el proceso de secado de brócoli ha sido documentado (Reyes et al., 2012; Mahn et al., 2016a y 2016b), la información sobre el efecto de las condiciones de secado sobre el contenido de sulforafano es más bien escasa. Reyes et al. (2012) estudiaron el secado de partículas de brócoli enriquecido en selenio, de tamaño homogéneo y forma definida, mediante un secador de lecho fluidizado pulsante. Los autores determinaron las condiciones de operación que maximizan la capacidad antioxidante y el contenido de selenio en el brócoli deshidratado. Mahn et al. (2016a) investigaron el efecto de las condiciones de secado en un secador de bandejas sobre la evolución del contenido de sulforafano en brócoli previamente enriquecido. Mahn et al. (2016b) investigaron la liofilización de brócoli y su efecto sobre el contenido de sulforafano en el producto final. Actualmente no existe información acerca del efecto de las condiciones de operación en secadores de lecho fluidizado pulsante sobre el contenido de sulforafano en brócoli.

El secado en lecho fluidizado se encuentra entre los métodos de secado más eficientes, debido al excelente contacto entre el aire de secado y las partículas, con elevados coeficientes de transferencia de calor y masa (Reyes et al., 2007). El tiempo de secado en este tipo de secador es menor que el tiempo requerido en secadores de bandeja o de túnel, lo que puede contribuir a reducir las pérdidas de diferentes compuestos nutricionales y bioactivos durante el secado de alimentos. Por lo tanto, el secado en lecho fluidizado parece una buena alternativa para deshidratar brócoli enriquecido en sulforafano. Por otra parte, el costo de operación de los secadores de lecho fluidizado es considerablemente menor que el de los liofilizadores, lo que representa una ventaja desde el punto de vista del proceso.

El desempeño de los secadores de lecho fluidizado se puede mejorar con algunas modificaciones al diseño, tales como el ingreso del aire de secado en forma de pulsos. Este tipo de modificación se conoce como secado en lecho fluidizado pulsante, el cual se logra con la ubicación de un disco perforado rotatorio debajo de la cama perforada que sostiene el lecho de partículas, provocando la fluidización intermitente del sólido en las zonas donde circula el aire (Reyes et al., 2008). El secador de lecho fluidizado pulsante facilita la fluidización de partículas que presentan una forma irregular, partículas frágiles o bien partículas con una distribución de tamaño heterogénea, y mejora la uniformidad de la fluidización reduciendo la formación de canales. Este tipo de secador también reduce los requerimientos de aire en un 30 a 50\% con respecto a un secador de lecho fluidizado convencional, y disminuye la caída de presión (Reyes et al., 2012). En el presente trabajo, previo al secado, el brócoli se sometió a un proceso que incluye la trituración del vegetal, obteniéndose partículas de tamaño heterogéneo y de forma irregular. Por lo tanto, el secado en lecho fluidizado pulsante sería adecuado en este caso.

El tamaño de las partículas y las propiedades del aire de secado, como son la humedad relativa, temperatura y velocidad, influyen en la pérdida de humedad del sólido, de ahí la importancia del estudio de la cinética de secado. Varias ecuaciones empíricas han sido propuestas para el estudio, modelado de la 
cinética y optimización del proceso de secado, como también para el diseño de secadores (Vega y Lemus, 2006). Entre estas ecuaciones se destacan las propuestas por Newton, Henderson-Pabis, Page, entre otros (Henderson, 1974; Page, 1949 y Aghbashlo et al., 2009).

El objetivo de este trabajo fue investigar el efecto de la temperatura del aire de secado y la velocidad de giro de la placa rotatoria en un secador de lecho fluidizado pulsante sobre el contenido de sulforafano y la cinética de secado de brócoli enriquecido en sulforafano. Las curvas de secado se ajustaron a modelos empíricos y se identificaron las condiciones de secado que maximizan el contenido de sulforafano en el producto deshidratado. La modelación del secado de brócoli en un lecho fluidizado pulsante contribuirá al diseño y escalamiento del proceso.

\section{METODOLOGÍA}

Se plantea el diseño experimental, se especifica la materia vegetal usada, se explica el proceso y el equipo de secado, se presenta la forma en que se determinó el contenido de sulforafano. Luego se presentan los resultados, ajuste a modelos empíricos y el análisis estadístico.

\section{Material vegetal}

Las cabezas de brócoli (Brassica oleracea var Italica cv. Avenger) de 3 días desde de la cosecha, fueron compradas en el mercado local (Santiago, Chile) a un solo proveedor. Las cabezas de brócoli fueron lavadas y cortadas en trozos de longitud de $5 \mathrm{~cm}$ y espesor de $0.7-0.9 \mathrm{~cm}$ (tallo). Previo al secado, el brócoli se sometió al proceso optimizado para maximizar el contenido de sulforafano (Pérez et al., 2014; Mahn y Pérez, 2016). Los trozos de brócoli se escaldaron en un baño termostático (Stuart, Reino Unido, Gran Bretaña) a $57^{\circ} \mathrm{C}$ durante 13 minutos. Inmediatamente después, los trozos de brócoli escaldado fueron sumergidos en un baño de agua - hielo. Posteriormente, el brócoli se trituró en presencia de $0.22 \mathrm{mg}$ de ácido ascórbico/g brócoli fresco, y finalmente la pasta obtenida se incubó en frascos cerrados herméticamente en un baño de agua termostático (Trilab, Ciudad de México, México) a $40^{\circ} \mathrm{C}$ durante $3 \mathrm{~h}$.

\section{Equipo}

El secador de lecho fluidizado pulsante (Fig. 1) corresponde al utilizado por Reyes et al. (2012). Fue fabricado en acero inoxidable, y consistió en una cámara de secado, un calefactor eléctrico (8000 W) controlable, un soplador centrífugo (10 HP), y un sistema de toma de muestra consistente en un ciclón con un filtro de bolsa como unidad de respaldo. La cámara de secado consiste en una columna cilíndrica de $1 \mathrm{~m}$ de alto conectada a una base de sección cónica truncada con un diámetro inferior de $0.25 \mathrm{~m}$ y un diámetro superior de $0.4 \mathrm{~m}$. El aire ingresa a la cámara de secado a través de una placa giratoria perforada con un $6 \%$ de superficie libre, la que permite la fluidización pulsada.

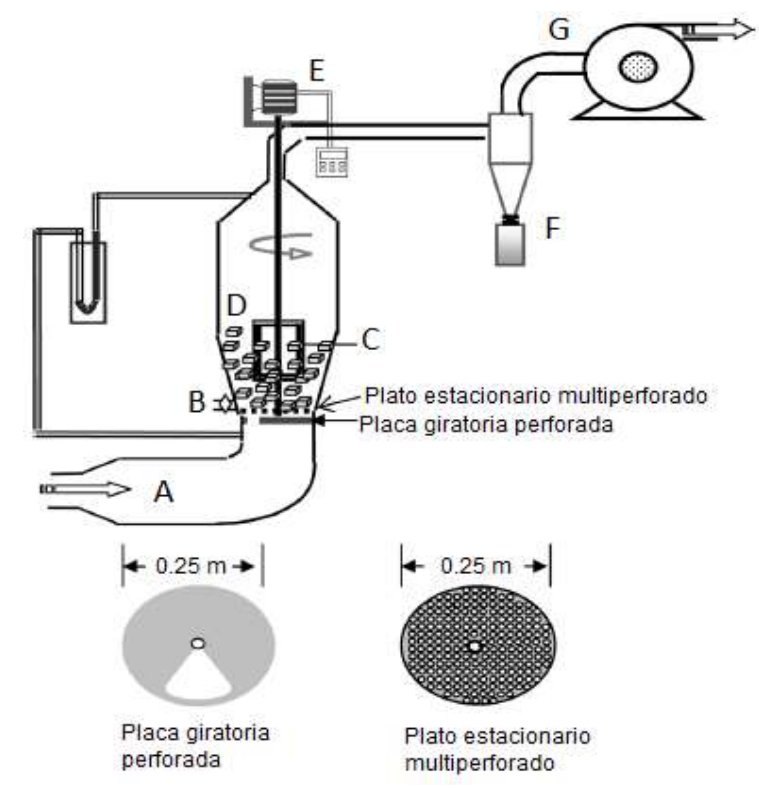

Fig. 1: Diagrama del secador de lecho fluidizado pulsante usado. A: calefactor, B: puerto de muestreo, C: ventana, D: cámara de fluidización, E: motor de frecuencia variable, F: ciclón, G: soplador centrífugo (adaptado de Reyes et al., 2012). 
El sistema de toma de muestra se ubicó en la sección inferior del secador, 0.07 m sobre la placa giratoria. Este sistema consistió en una cuchara de muestreo de $30 \mathrm{~mL}$ que se introduce a través de un puerto de muestreo, de manera que es posible tomar muestras en distintas posiciones sobre el plano horizontal dentro del lecho. Una vez tomada la muestra, la cuchara era retirada y el puerto, cerrado. La salida de sólidos a través de la placa giratoria se evitó localizando una malla fina sobre ella. El giro de la placa rotatoria se logró mediante un eje vertical movido por un motor eléctrico (1/4 HP) provisto de un regulador de frecuencia. La velocidad de flujo de aire a la entrada de la cámara de secado se midió con un anemómetro de veleta manual (precisión de $0.1 \mathrm{~m} / \mathrm{s}$ ) en un ducto de $0.10 \mathrm{~m}$ de diámetro. La temperatura dentro de la cámara de secado se midió con un termopar tipo $\mathrm{K}$ (precisión de $0.1^{\circ} \mathrm{C}$ ).

\section{Cinética de Secado}

En cada corrida de secado se utilizaron $2 \mathrm{~kg}$ de pasta de brócoli incubado. Para determinar la cinética de secado se tomaron muestras de partículas desde el interior del lecho a intervalos de 2 minutos para los primeros 14 minutos y cada 4 minutos durante los siguientes 20 minutos, siguiendo la metodología reportada por Reyes et al. (2012). Cuando una corrida experimental excedía este tiempo, las siguientes muestras eran tomadas a intervalos de 8 minutos hasta alcanzar el peso constante. El contenido de humedad de estas muestras se determinó de acuerdo con el método AOAC 920.151 por secado a 80 ㄷ en un horno a vacío (Cole \& Palmer, modelo 60061 Holdpack, Illinois) hasta alcanzar un peso constante, y las mediciones fueron realizadas en triplicado. El contenido de humedad se expresó en base seca.

Las curvas de cinéticas de secado se representaron como la razón de humedad adimensional (MR), definida en la Ec. 1, frente al tiempo de secado (t), para cada corrida experimental. En la Ec. 1, $M_{t}$ es la humedad en función del tiempo y $M_{0}$ es la humedad inicial. A partir de las curvas de secado se determinó la humedad crítica $\left(\mathrm{M}_{\mathrm{cr}}\right)$ y el tiempo en que ésta fue alcanzada en cada caso. Las cinéticas de secado se ajustaron a cuatro modelos empíricos ampliamente utilizados en alimentos, descritos en la Tabla 1. La calidad del ajuste de las curvas de secado a los modelos empíricos se evaluó mediante el coeficiente de determinación $\left(R^{2}\right)$ y el estadístico chi-cuadrado $\left(X^{2}\right)$. Los ajustes se realizaron con el programa OriginPro 9.0 (OriginLab Corp., USA).

$M R=\frac{M_{t}}{M_{0}}$

Tabla1: Modelos matemáticos seleccionados para describir la cinética de secado del brócoli triturado

\begin{tabular}{lll}
\hline Nombre del modelo & Ecuación del Modelo & Referencias \\
\hline Newton & $\mathrm{MR}=\exp \left(-K_{N} \cdot \mathrm{t}\right)$ & Henderson, 1974 \\
Page & $\mathrm{MR}=\exp \left(-K_{P} \cdot \mathrm{t}^{\mathrm{n}}\right)$ & Page, 1949 \\
Henderson y Pabis & $\mathrm{MR}=\mathrm{a} \exp \left(-K_{H} \cdot \mathrm{t}\right)$ & Aghbashlo et al., 2009 \\
Logarítmico & $\mathrm{MR}=\mathrm{b} \exp \left(-K_{l} \cdot \mathrm{t}\right)+\mathrm{c}$ & Karathanos, 1999 \\
\hline
\end{tabular}

\section{Diseño experimental}

Para la optimización del proceso de secado se utilizó un diseño central compuesto de precisión uniforme, con dos factores experimentales: temperatura del aire de secado (35 y 65으 y velocidad de giro de la placa rotatoria (60 y $100 \mathrm{rpm})$, cuatro puntos axiales y cinco puntos centrales. La matriz experimental se muestra en la Tabla 2. Para determinar los niveles de los factores experimentales, se realizaron ensayos preliminares para estudiar la fluido dinámica en el sistema con partículas de brócoli, a velocidades de flujo de aire de 0.5, 1, 1.5 y $2(\mathrm{~m} / \mathrm{s})$, los que mostraron que a $1.5 \mathrm{~m} / \mathrm{s}$ se observa la mejor calidad de fluidización, ya que a velocidades superiores se incrementa el arrastre de partículas fuera del lecho y a velocidades bajo $1 \mathrm{~m} / \mathrm{s}$, la fluidización es parcial.

\section{Determinación del contenido de Sulforafano}

El contenido de sulforafano se determinó mediante HPLC de fase reversa, según el método propuesto por Liang et al. (2006), con algunas modificaciones (Pérez et al., 2014). Se utilizó un equipo HPLC-DAD (Shimadzu, Kyoto, Japan) y una columna C18 (tamaño de partícula $5 \mu \mathrm{m}, 250 \times 4.6 \mathrm{~mm}$ ) (Agilent Technologies, Santa Clara, CA, E.E.U.U.). Se pesó 1 gramo de cada una de las muestras de brócoli, se extrajo en $10 \mathrm{~mL}$ de cloruro de metileno adicionando con $0.5 \mathrm{~g}$ de $\mathrm{Na}_{2} \mathrm{SO}_{4}$ anhidro. La mezcla se filtró con papel filtro para separar residuos vegetales y el remanente de cloruro de metileno se secó a $30^{\circ} \mathrm{C}$ bajo vacío en un evaporador rotatorio (modelo RE300, Stuart). Luego el residuo se disolvió en $1 \mathrm{~mL}$ de acetonitrilo y se filtró con un filtro de jeringa de 0.22 micras, para finalmente inyectarlo en el HPLC. Los reactivos (calidad HPLC) fueron adquiridos a Sigma-Aldrich (Schnelldorf, Alemania). 


\section{Análisis estadístico}

El efecto de los factores experimentales sobre el contenido de sulforafano se determinó mediante la metodología de superficie de respuesta (Zambrano et al., 2007; Mazo et al., 2008), utilizando un modelo polinomial de segundo orden a fin de describir el comportamiento experimental (Ec. 2). En la Ec. 2, ŷ es el valor predicho de la respuesta; $\beta_{0}, \beta_{i}, \beta_{i i}$, y $\beta_{i j}$ son los coeficientes de regresión para la intersección, efectos lineales, cuadráticos y la interacción, respectivamente; $\mathrm{k}$ es el número de parámetros independientes ( $\mathrm{k}=$ 2), y $X_{i}, X_{j}$ son los niveles codificados de los factores experimentales. Se consideró un $95 \%$ de confianza. Se utilizó el programa JMP 9.0.1 (SAS Institute Inc., U.S.A.). El modelo de regresión se utilizó para determinar las condiciones óptimas de secado que maximizan el contenido de sulforafano. Se ejecutaron corridas experimentales usando las condiciones óptimas predichas para validar el modelo.

$\hat{\mathrm{y}}=\beta_{0}+\sum_{i=1}^{k} \beta_{i} X_{i}+\sum_{i=1}^{k} \beta_{i i} X_{i}^{2}+\sum \sum_{i<j=1}^{k} \beta_{i j} X_{i} X_{j}$

\section{RESULTADOS Y DISCUSION}

Se presentan los resultados de la cinética de secado, se discuten el modelado matemático de las curvas de secado, se analizan los resultados sobre contenido de sulforafano y finalmente se discute la optimización del proceso de secado por lecho fluidizado pulsante.

\section{Contenido de sulforafano}

El contenido de sulforafano en el brócoli sometido a las diferentes corridas de secado se presenta en la Tabla 2. El proceso de secado generó pérdidas importantes del compuesto en todas las condiciones de secado estudiadas, en comparación con el contenido del brócoli enriquecido en sulforafano (sometido a escaldado e incubado), a pesar de haberse utilizado temperaturas del aire de secado relativamente bajas $\left(29\right.$ a $\left.71^{\circ} \mathrm{C}\right)$. La menor pérdida del compuesto se presentó utilizando una temperatura del aire de secado igual a $35^{\circ} \mathrm{C}$ y una velocidad de giro de la placa igual a $100 \mathrm{rpm}$ (T2), donde se logró retener el $17 \%$ del contenido de inicial sulforafano presente en el brócoli pre-procesado. Este valor corresponde a $1.4 \mu \mathrm{mol} / \mathrm{g}$ en base seca, siendo superior al contenido de sulforafano en el brócoli fresco sin enriquecer $(1.1 \mu \mathrm{mol} / \mathrm{g}$ en base seca).

La pérdida del contenido de sulforafano durante el secado ha sido reportada por Tanongkankit et al. (2011), quienes estudiaron la evolución de sulforafano en hojas exteriores de repollo durante el proceso de secado en un secador de bandeja con una velocidad de aire de $2 \mathrm{~m} / \mathrm{s}$ a diferentes temperaturas $\left(50,60\right.$ y $\left.70{ }^{\circ} \mathrm{C}\right)$. Los investigadores reportaron que aproximadamente el $90 \%$ del sulforafano sufrió degradación térmica durante el proceso. Basados en esa investigación, Lekcharoenkul et al. (2014) estudiaron el efecto del cambio gradual de la temperatura durante el secado con aire caliente, secado al vacío y secado híbrido (secado a baja presión con vapor sobrecalentado seguido por secado al vacío), sobre la evolución de sulforafano para la misma variedad vegetal. Encontraron un comportamiento similar al reportado por Tanongkankit et al. (2011), detectando una pérdida de sulforafano superior al $90 \%$ con respecto al contenido inicial. En ambos estudios los autores atribuyeron la pérdida de sulforafano a la temperatura y al tiempo de secado prolongado. Contrario a estos resultados, Mahn et al. (2016a) reportaron una retención del $54 \%$ de sulforafano en el brócoli triturado luego de ser sometido a secado convectivo en un secador de bandejas a $70^{\circ} \mathrm{C}$ con una velocidad de secado de $2 \mathrm{~m} / \mathrm{s}$ por $4 \mathrm{~h}$. Esta retención representó un valor 4 veces mayor que el contenido de sulforafano en el brócoli fresco. Estos resultados difieren de los reportados por Tanongkankit et al. (2011) y Lekcharoenkul et al. (2014) debido probablemente a que los autores utilizaron hojas de repollo, y no inflorescencias de brócoli. Ambas matrices vegetales presentan estructuras muy diferentes, lo que se traduce en distintos comportamientos durante el secado.

La degradación térmica del sulforafano no sería la responsable de las pérdidas observadas en este trabajo, ya que se utilizaron temperaturas del aire de secado relativamente bajas y periodos de secado comparativamente breves, con la temperatura del brócoli bajo los $40^{\circ} \mathrm{C}$, temperatura a la cual comienza la degradación térmica del sulforafano. Una posible explicación a la pérdida aparente de sulforafano es la reacción entre sulforafano y compuestos que contienen grupos tiol presentes en brócoli, formando ditiocarbamatos en una reacción de óxido reducción reversible (Shibata et al., 2011). Sin embargo, esta hipótesis debe ser probada en un futuro. Otra posible explicación es el arrastre del compuesto en el aire de secado, dada la configuración y operación del equipo el contacto aire-partícula se maximiza y por lo tanto se facilita el arrastre del compuesto, en comparación con otros métodos de secado estudiados tales como secador de bandejas y liofilización. 


\section{Cinética de secado}

El contenido promedio de humedad inicial del brócoli pre-procesado (escaldado e incubado) fue de $8.85 \mathrm{~kg}$ $\mathrm{H}_{2} \mathrm{O} / \mathrm{kg}$ base seca. Las variaciones de la razón de humedad (MR) con el tiempo de secado en las diferentes condiciones de secado y el ajuste a los modelos cinéticos se muestran en la Fig. (2). Las cinéticas muestran una relación inversa entre la temperatura del aire de secado y la velocidad de giro de la placa rotatoria con el tiempo de secado final necesario para alcanzar una razón de humedad de 0.019. Los niveles altos de temperatura y de velocidad de giro produjeron tiempos de secado más cortos. Los valores del contenido de humedad crítica en base seca $\left(\mathrm{M}_{\mathrm{rr}}\right)$ fueron determinados desde la pendiente de las curvas de secado para cada corrida. Los valores obtenidos fluctuaron entre 0.44 y $0.72 \mathrm{~kg} \mathrm{H} \mathrm{H}_{2} \mathrm{O} / \mathrm{kg}$ base seca. Los datos de humedad crítica y el tiempo en que esta fue alcanzada se informan en la Tabla 2.

Tabla 2: Contenido de sulforafano en cada corrida de secado, brócoli fresco y brócoli enriquecido humedad crítica y tiempo para alcanzar la humedad crítica en cada corrida experimental.

\begin{tabular}{|c|c|c|c|c|c|}
\hline Corrida & Temperatura $\left[{ }^{\circ} \mathrm{C}\right]$ & Velocidad de giro [rpm] & Sulforafano $[\mu \mathrm{mol} / \mathrm{g}$ b.s $]$ & $M_{c r}$ & Tiempo [min] \\
\hline T1 & 65 & 100 & $0.9 \pm 0.0$ & $0.50 \pm 0.02$ & 2.5 \\
\hline T2 & 35 & 100 & $1.4 \pm 0.1$ & $0.58 \pm 0.02$ & 5.0 \\
\hline T3 & 50 & 80 & $0.9 \pm 0.1$ & $0.59 \pm 0.03$ & 5.0 \\
\hline T3 & 50 & 80 & $0.9 \pm 0.3$ & $0.60 \pm 0.02$ & 5.1 \\
\hline T3 & 50 & 80 & $1.0 \pm 0.1$ & $0.57 \pm 0.02$ & 5.0 \\
\hline T3 & 50 & 80 & $1.0 \pm 0.0$ & $0.59 \pm 0.01$ & 5.0 \\
\hline T3 & 50 & 80 & $0.9 \pm 0.1$ & $0.59 \pm 0.04$ & 5.0 \\
\hline T4 & 65 & 60 & $0.7 \pm 0.0$ & $0.55 \pm 0.01$ & 3.0 \\
\hline T5 & 35 & 60 & $0.7 \pm 0.0$ & $0.65 \pm 0.02$ & 5.0 \\
\hline T6 & 50 & 108 & $1.1 \pm 0.1$ & $0.63 \pm 0.01$ & 4.2 \\
\hline T7 & 71 & 80 & $0.5 \pm 0.1$ & $0.50 \pm 0.02$ & 3.0 \\
\hline T8 & 29 & 80 & $0.9 \pm 0.0$ & $0.72 \pm 0.04$ & 7.5 \\
\hline T9 & 50 & 52 & $0.8 \pm 0.1$ & $0.44 \pm 0.04$ & 8.3 \\
\hline \multicolumn{3}{|c|}{ Contenido de sulforafano en el brócoli fresco } & $1.1 \pm 0.2$ & & \\
\hline \multicolumn{3}{|c|}{$\begin{array}{l}\text { Contenido de sulforafano en brócoli enriquecido previo al } \\
\text { secado }\end{array}$} & $8.1 \pm 0.3$ & & \\
\hline
\end{tabular}

El reducido tiempo de secado especialmente a las temperaturas de 65 y $71^{\circ} \mathrm{C}$, así como el corto tiempo necesario para alcanzar la humedad critica a estas temperaturas, se debe al aumento del gradiente de temperatura entre el aire de secado y el brócoli triturado, lo que constituye el potencial para la transferencia de calor, favoreciendo la rápida evaporación del agua. Por otro lado, la disminución del tiempo de secado final, provocado por el aumento de la velocidad de giro de la placa, se puede atribuir al movimiento en sentido axial y radial del aire de secado, resultando en una trayectoria más extensa del gas dentro del lecho (Reyes et al., 2012). Esto se traduce finalmente en un mejor contacto entre las partículas de brócoli y el aire de secado.

La marcada disminución del contenido de humedad durante el periodo de velocidad de secado constante se debe a que el agua libre en la superficie del brócoli es arrastrada con facilidad por el aire que produce la fluidización. Se debe tener presente que el agua eliminada en este periodo no es sólo agua superficial, ya que también proviene del interior del brócoli. Esto corresponde al comportamiento típico de materiales porosos, tales como el brócoli (Mulet et al., 1999). Una vez finalizado el periodo de secado a velocidad constante, se observó una menor pérdida de humedad, atribuible a barreras difusionales.

El análisis estadístico mostró que la temperatura del aire de secado tuvo un efecto significativo negativo sobre $M_{c r}(p=0.0202)$ y sobre el tiempo de secado $(p=0.0183)$, tal como se muestra en la Fig. (3). Esto significa que a medida que aumenta la temperatura, el contenido de humedad crítica y el tiempo necesario para alcanzarla disminuyen. Esto indica que a niveles altos de temperatura $\left(65-71^{\circ} \mathrm{C}\right)$ la disminución de humedad en el periodo de secado a velocidad constante ocurre más rápido. A pesar que el periodo de velocidad constante para todas las corridas resultó ser relativamente breve $(2.5-8.3 \mathrm{~min})$, fue en este periodo donde se presentó la mayor eliminación del contenido de humedad. Después de alcanzada la humedad crítica, la velocidad de secado fue menor, etapa que corresponde al periodo de velocidad de secado decreciente. 


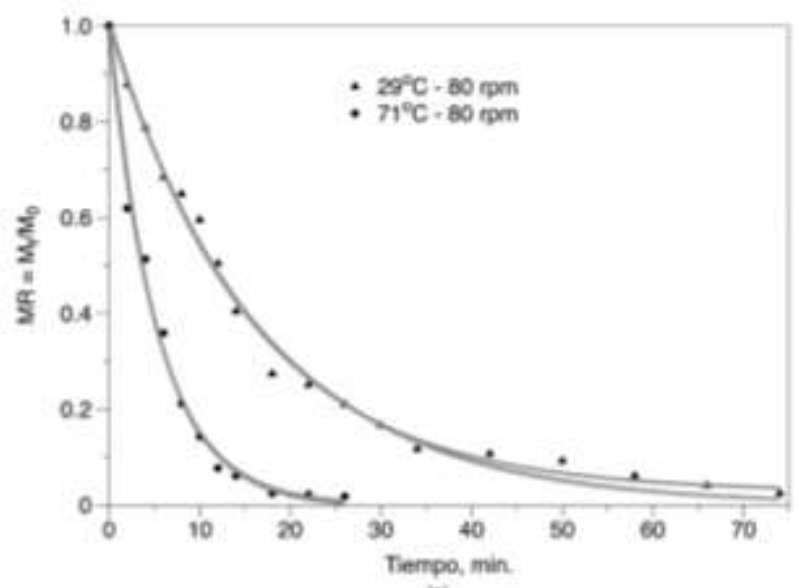

(a)

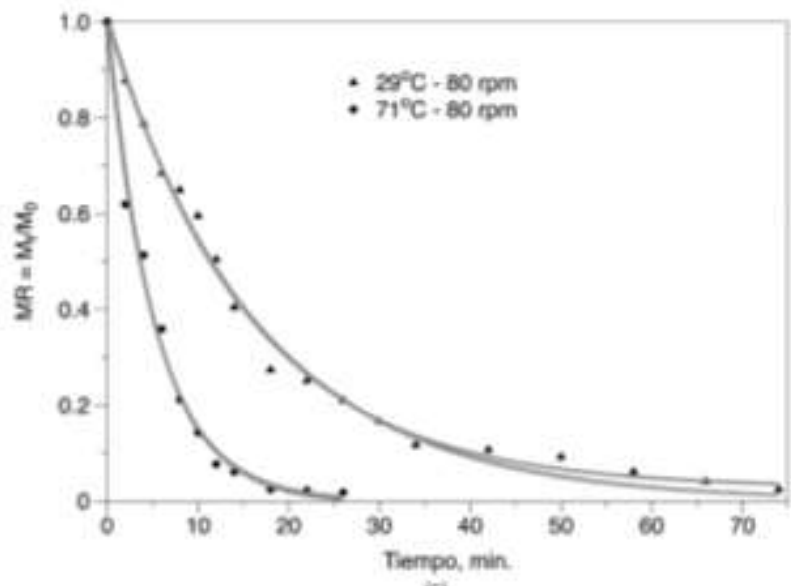

(a)
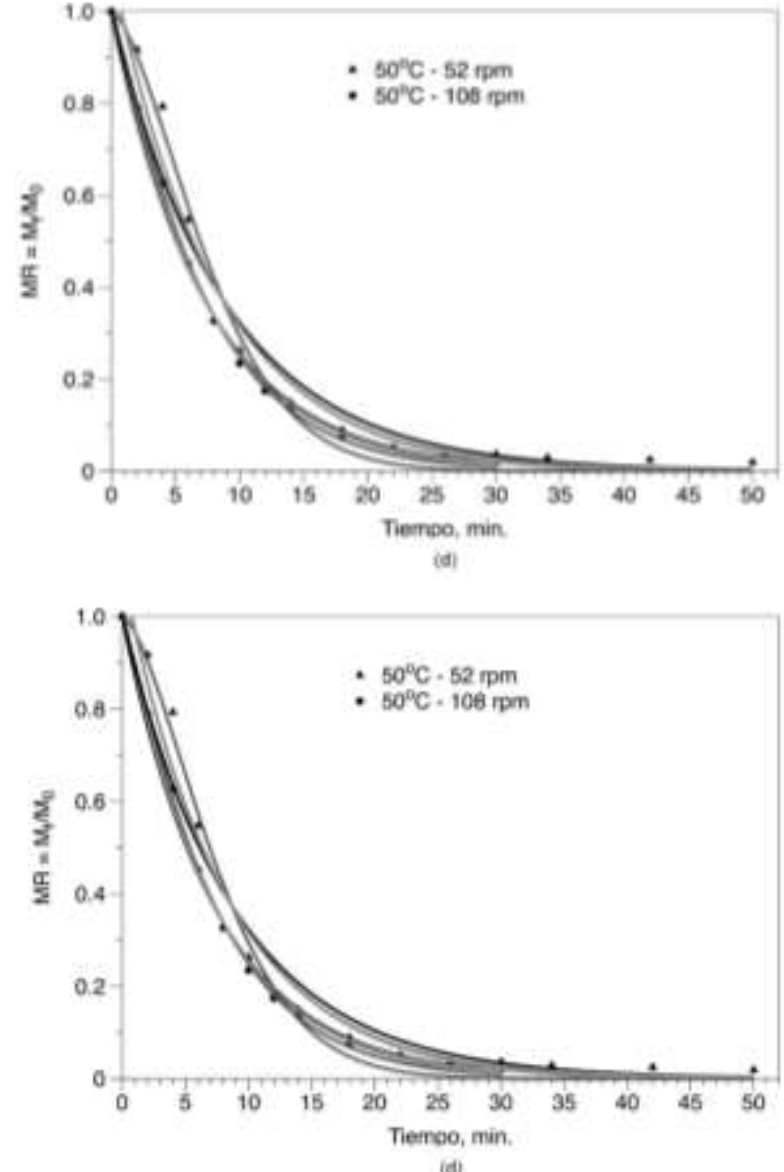

(d)

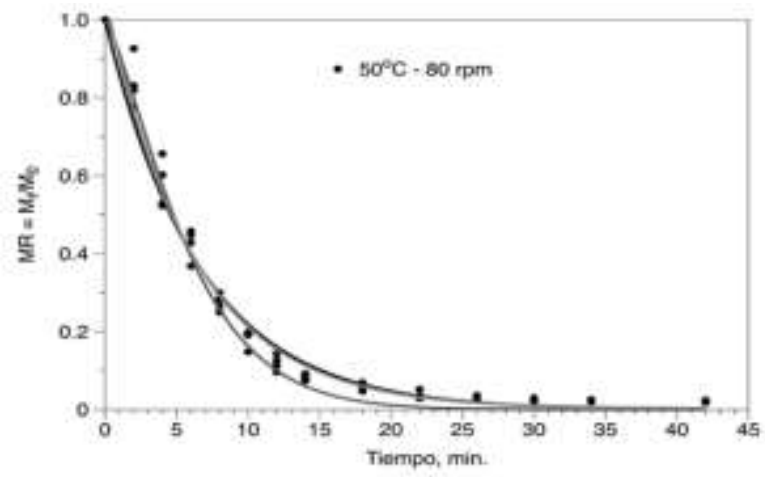

Fig. 2: Cinética de secado para las distintas corridas y ajuste a los modelos cinéticos de Newton (—), Page (—), Henderson y Pabis (-) y Logarítmico (-).

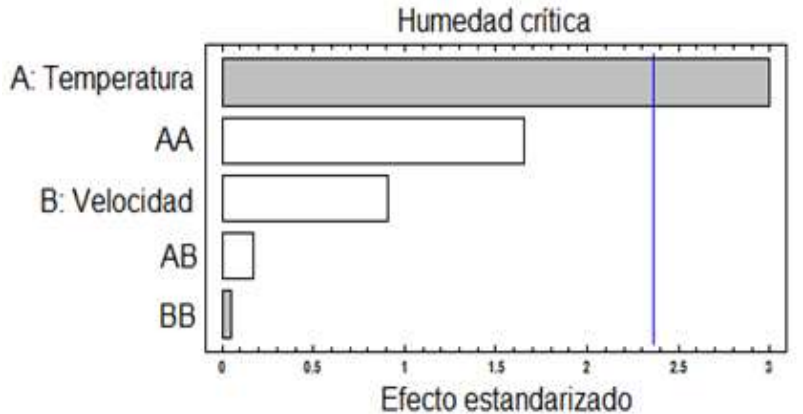

(a)

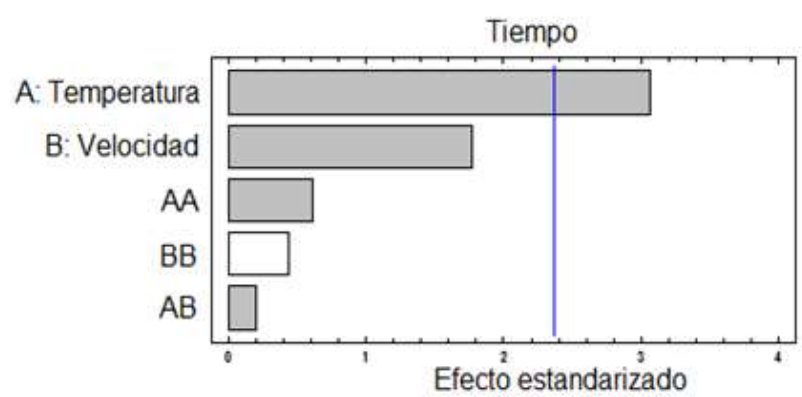

(b)

Fig. 3: Gráficos de Pareto para el efecto de temperatura y velocidad de rotación de la placa giratoria sobre (a) humedad crítica y (b) tiempo en que se alcanza la humedad crítica. Las barras blancas indican efecto positivo y las grises indican efecto negativo. 


\section{Ajuste de las curvas de secado a modelos empíricos}

Las curvas de secado se ajustaron a los modelos empíricos descritos en la Tabla 1. El ajuste de los modelos cinéticos a los datos experimentales se presenta en la Fig. (2). En la Tabla 3 se presentan los valores de los parámetros cinéticos obtenidos para cada modelo y los parámetros estadísticos indicativos de la calidad de los ajustes. Todos los modelos estudiados presentaron un buen ajuste a los datos experimentales, donde los valores de $\mathrm{R}^{2}$ variaron entre $95.1 \%$ y $99.9 \%$, mientras que los valores de $\chi^{2}$ variaron entre $0.07 \cdot 10^{-3}$ y $1.03 \cdot 10^{-3}$. El modelo de Page entregó el mejor ajuste a los datos experimentales, con $\mathrm{R}^{2} \geq 99 \%$. Este modelo ha sido aplicado con buenos resultados por varios investigadores en la descripción de la cinética de secado de vegetales (Vega y Lemus, 2006; Ah-Hen et al., 2011; Reyes et al., 2008). Teniendo en cuenta que el modelado de la cinética tiene como objetivo predecir la velocidad y el tiempo de secado, el modelo de Page se podría utilizar para simular cinéticas de secado de brócoli triturado. Este modelo también ofrecería utilidad para el escalamiento del proceso.

Se realizó un análisis de varianza (ANOVA) con un 95\% de confianza a los parámetros de cada modelo, a objeto de determinar el efecto de las condiciones de secado sobre ellos. La temperatura tuvo un efecto positivo significativo sobre $\mathrm{K}_{\mathrm{j}}(\mathrm{j}=\mathrm{N}, \mathrm{P}, \mathrm{H}, \mathrm{l})$; esto significa que el aumento de la temperatura del aire provocó el incremento del valor de este parámetro en toda la región experimental, para todos los modelos. La dependencia del parámetro cinético $\mathrm{K}_{\mathrm{j}}$ con la temperatura, ha sido reportada para el secado convectivo de papaya (Lemus-Mondaca et al., 2009 y Vega y Lemus, 2006), pepino (Vega-Galvez et al., 2008) y pimiento rojo (Uribe et al., 2011). Los investigadores sugirieron que el parámetro $\mathrm{K}_{\mathrm{j}}$ es directamente proporcional al coeficiente de transferencia de masa, luego de relacionarlo con el coeficiente de difusividad del agua a través de una ecuación tipo Arrhenius.

El parámetro 'a' del modelo de Henderson-Pabis, fue afectado negativamente por la interacción de temperatura consigo misma, es decir este parámetro presentó un incremento al aumentar la temperatura de secado hasta el nivel medio, donde alcanza su valor máximo, pero al sobrepasar esta temperatura disminuye su valor. En el secado convectivo de papaya, Vega y Lemus (2006) informaron que este parámetro no se vio afectado por la temperatura. Un comportamiento similar fue reportado en el secado de murta por Ah-Hen et al. (2011), quienes informaron que el parámetro no fue afectado por la temperatura y los procesos de secado (vacío y atmosférico). Por su parte Babalis y Belessiotis, (2004), observaron la dependencia lineal del parámetro 'a' al aumentar la velocidad del aire de 0.5 a $3.0 \mathrm{~m} \cdot \mathrm{s}^{-1}$, en el secado de higos.

Los parámetros $\mathrm{n}$ (modelo de Page), b y c (modelo Logarítmico) no fueron afectados por los factores experimentales. Ah-Hen et al. (2011), informaron que los parámetro n y b se mantuvieron constantes, y el c aumentó con el nivel más alto de temperatura $\left(70^{\circ} \mathrm{C}\right)$ durante el secado a vacío y atmosférico de la murta. Senadeera et al. (2003) estableció que el parámetro n permanecía constante con la variación de la temperatura, en el secado de frijoles, patatas y peras. Karanthanos y Belessiotis (1999) deshidrataron frutas con y sin piel, y reportaron que el parámetro $\mathrm{n}$ aumentó con la presencia de piel dependiendo del espesor de la misma y del tipo de producto a secar. La diferencia en el comportamiento de los parámetros a, n, b y c puede ser debido a su importante dependencia de las características estructurales del tejido vegetal.

\section{Maximización del contenido de sulforafano}

El efecto de los factores experimentales sobre el contenido de sulforafano se presenta en la Fig. (4) mediante un gráfico de Pareto. La velocidad de giro de la placa tuvo un efecto significativo positivo sobre el contenido de sulforafano $(p=0.0012)$, mientras que la temperatura, su interacción de segundo orden y la interacción entre temperatura y velocidad de giro, presentaron efectos significativos negativos, con valores $p$ iguales a $0.0060,0.0420$ y 0.0160 , respectivamente. Esto significa que un aumento en la velocidad de giro, desde el nivel inferior $(60 \mathrm{rpm})$ al superior $(100 \mathrm{rpm})$ produce menores pérdidas de sulforafano en el producto deshidratado. Además, al aumentar la temperatura desde $35^{\circ} \mathrm{C}$ a $65^{\circ} \mathrm{C}$ se incrementan las pérdidas del compuesto. Por lo tanto, las pérdidas de sulforafano se minimizan al utilizar temperatura del aire de secado baja y velocidad de giro de la placa alta.

El modelo de regresión que describe el contenido de sulforafano como una función de los factores experimentales, en niveles codificados, se muestra en la Ec. (3). Sólo las variables que tuvieron efecto significativo sobre la respuesta fueron incluidas en el modelo. En la Ec. (3), [S] es el contenido de sulforafano, A es la temperatura de secado del aire y B es la velocidad de giro de la placa rotatoria. El modelo de regresión fue capaz de explicar el $89.4 \%$ de la variabilidad en la respuesta, lo que indica que el modelo representa adecuadamente el comportamiento del sistema.

$[S]=0.944-0.238 \cdot A+0.321 \cdot B-0.163 \cdot A^{2}-0.274 \cdot A \cdot B$ 


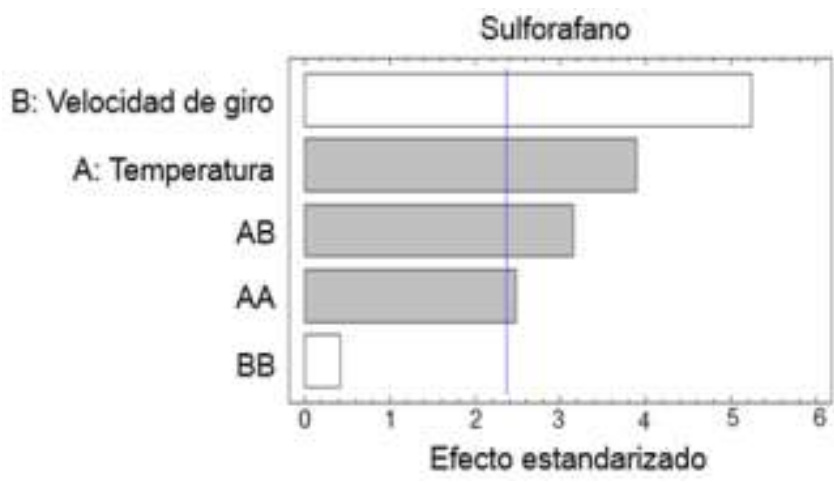

Fig. 4: Efecto de la temperatura y la velocidad de giro de la placa rotatoria sobre el contenido de sulforafano. Las barras blancas indican efecto positivo y las grises indican efecto negativo.

Tabla 3: Parámetros de los modelos cinéticos y análisis estadístico del ajuste de los modelos. $\mathrm{K}_{\mathrm{j}}$ representa las constantes $\mathrm{K}_{\mathrm{N}}, \mathrm{K}_{\mathrm{P}}, \mathrm{K}_{\mathrm{H}}$ y $\mathrm{K}_{\mathrm{I}}$ del modelo que corresponde. Las condiciones experimentales correspondientes a cada corrida se muestran en la Tabla 1.

\begin{tabular}{|c|c|c|c|c|c|c|c|c|}
\hline \multirow{2}{*}{ Corrida } & \multirow{2}{*}{ Modelos } & \multicolumn{5}{|c|}{ Parámetros } & \multirow{2}{*}{$R^{2} \%$} & \multirow{2}{*}{$\chi^{2}\left(\cdot 10^{3}\right)$} \\
\hline & & $\mathrm{K}_{\mathrm{j}}$ & $\mathrm{n}$ & $a$ & $\mathrm{~b}$ & $\mathrm{C}$ & & \\
\hline \multirow{4}{*}{$\mathrm{T} 1$} & Newton & 0.25143 & - & - & - & - & 95.1 & 4.40 \\
\hline & Page & 0.45552 & 0.64709 & & & & 98.9 & 1.11 \\
\hline & Henderson & 0.23244 & - & 0.93337 & - & - & 95.7 & 4.35 \\
\hline & Logarítmico & 0.29199 & - & - & 0.90521 & 0.05551 & 96.6 & 3.94 \\
\hline \multirow{4}{*}{$\mathrm{T} 2$} & Newton & 0.11015 & - & - & - & - & 99.8 & 0.23 \\
\hline & Page & 0.10805 & 1.00842 & - & - & - & 99.8 & 0.26 \\
\hline & Henderson & 0.11001 & - & 0.99885 & - & - & 99.8 & 0.26 \\
\hline & Logarítmico & 0.10618 & - & - & 1.00792 & -0.01347 & 99.8 & 0.25 \\
\hline \multirow{4}{*}{ T3 } & Newton & 0.12757 & - & - & - & - & 98.8 & 1.03 \\
\hline & Page & 0.09142 & 1.29780 & & & & 99.5 & 0.51 \\
\hline & Henderson & 0.12072 & - & 0.95261 & - & - & 99.1 & 0.83 \\
\hline & Logarítmico & 0.12711 & - & - & 0.94246 & 0.01684 & 99.2 & 0.83 \\
\hline \multirow{4}{*}{$\mathrm{T} 4$} & Newton & 0.22154 & - & - & - & - & 98.9 & 0.95 \\
\hline & Page & 0.32025 & 0.78750 & - & - & - & 99.9 & 0.07 \\
\hline & Henderson & 0.21336 & - & 0.96492 & - & - & 99.1 & 0.90 \\
\hline & Logarítmico & 0.24088 & - & - & 0.94413 & 0.03489 & 99.6 & 0.46 \\
\hline \multirow{4}{*}{$\mathrm{T} 5$} & Newton & 0.09571 & - & - & - & - & 99.2 & 0.74 \\
\hline & Page & 0.12040 & 0.90287 & - & - & - & 99.4 & 0.56 \\
\hline & Henderson & 0.09352 & - & 0.98122 & - & - & 99.2 & 0.75 \\
\hline & Logarítmico & 0.10370 & - & - & 0.96207 & 0.03416 & 99.2 & 4.28 \\
\hline \multirow{4}{*}{ T6 } & Newton & 0.13590 & - & - & - & - & 99.6 & 0.40 \\
\hline & Page & 0.10691 & 1.11481 & - & - & - & 99.9 & 0.15 \\
\hline & Henderson & 0.13930 & - & 1.02450 & - & - & 99.7 & 0.35 \\
\hline & Logarítmico & 0.13690 & - & - & 1.02931 & -0.00690 & 99.7 & 0.38 \\
\hline \multirow{4}{*}{$\mathrm{T} 7$} & Newton & 0.18950 & - & - & - & - & 99.1 & 0.88 \\
\hline & Page & 0.19840 & 0.97570 & - & - & - & 99.1 & 0.97 \\
\hline & Henderson & 0.18690 & - & 0.98590 & & & 99.2 & 0.95 \\
\hline & Logarítmico & 0.18400 & - & - & 0.98970 & -0.00540 & 99.2 & 1.06 \\
\hline \multirow{4}{*}{$\mathrm{T} 8$} & Newton & 0.06000 & - & - & - & - & 99.2 & 0.77 \\
\hline & Page & 0.06160 & 0.99052 & - & - & - & 99.2 & 0.82 \\
\hline & Henderson & 0.06000 & - & 0.99982 & - & - & 99.2 & 0.82 \\
\hline & Logarítmico & 0.06471 & - & - & 0.98251 & 0.02771 & 99.4 & 0.72 \\
\hline \multirow{4}{*}{ T9 } & Newton & 0.11321 & - & - & - & - & 96.7 & 4.06 \\
\hline & Page & 0.03462 & 1.54855 & - & - & - & 99.3 & 0.94 \\
\hline & Henderson & 0.12450 & - & 1.09820 & - & - & 97.6 & 3.16 \\
\hline & Logarítmico & 0.12250 & - & - & 1.10340 & -0.00720 & 97.6 & 3.41 \\
\hline
\end{tabular}


La Fig. (5) muestra el diagrama de superficie de respuesta para sulforafano, en donde se observa que el contenido de sulforafano se ve favorecido a bajas temperaturas de secado y a altas velocidades de giro de la placa rotatoria. La combinación de altas velocidades de giro con las altas temperaturas provoca una disminución en esta respuesta. En la Fig. (5) se observa que el mayor valor de sulforafano coincide con uno de los vértices del espacio experimental, por lo tanto no es posible asegurar que efectivamente se trate de un máximo. Las condiciones de secado que entregan el mayor contenido de sulforafano en brócoli corresponden a temperatura del aire de secado igual a $29^{\circ} \mathrm{C}$ y velocidad de giro de la placa igual a $108 \mathrm{rpm}$. En estas condiciones, el contenido de sulforafano predicho por el modelo fue $1.5 \mu \mathrm{mol} / \mathrm{g}$ base seca. Para verificar si se trata de un máximo sería necesario ejecutar corridas de secado a temperatura del aire de secado inferior a $29^{\circ} \mathrm{C}$, lo que resulta impracticable dadas las características del equipo usado en este estudio.

La verificación experimental de la predicción del modelo entregó un contenido de sulforafano en el brócoli deshidratado igual a $1.5 \pm 0.1 \mu \mathrm{mol} / \mathrm{g}$ base seca, en las condiciones que maximizaron el contenido de sulforafano. Este valor representa un incremento del $25 \%$ con relación al brócoli fresco y una disminución del $81.5 \%$ con respecto al brócoli enriquecido en sulforafano. Los resultados expuestos en este trabajo podrían ser útiles para diseñar un proceso de secado mediante fluidización pulsante que permita minimizar las pérdidas de sulforafano.

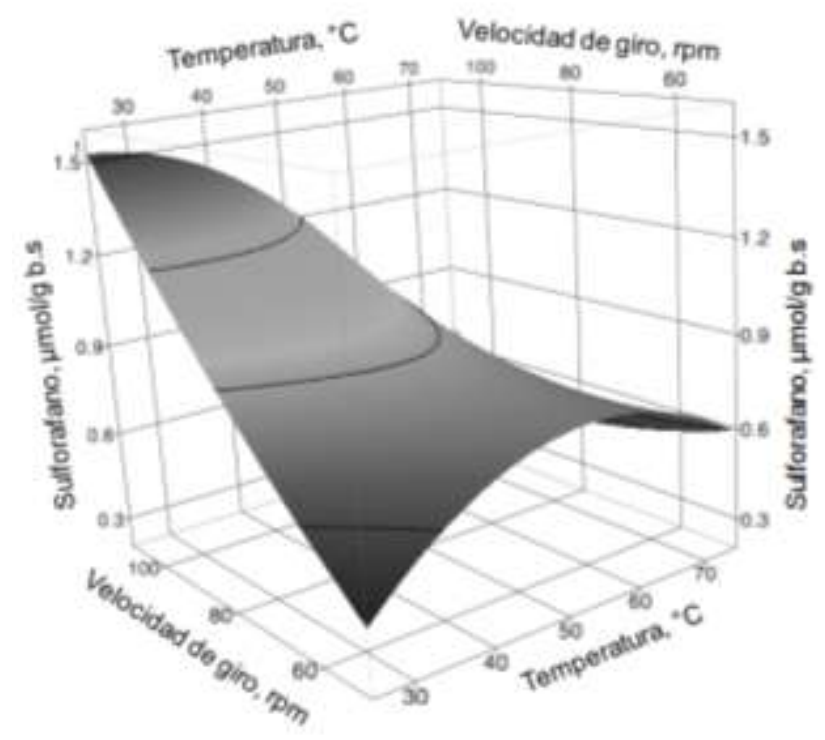

Fig. 5: Superficie de respuesta para el sulforafano frente a la temperatura y velocidad de giro.

\section{CONCLUSIONES}

La humedad crítica fluctuó entre 0.44 y $0.72 \mathrm{~kg} \mathrm{H}_{2} \mathrm{O} / \mathrm{kg}$ base seca en las diferentes corridas, y fue alcanzada después de 2.5 a 8.3 min de secado, dependiendo de las condiciones de operación. La temperatura del aire de secado tuvo un efecto significativo negativo sobre la humedad crítica. La velocidad de giro de la placa rotatoria no afectó significativamente la cinética de secado. Todos los modelos de cinética de secado estudiados entregaron ajustes satisfactorios a las curvas $\left(R^{2}>95 \%\right.$ y $\left.x^{2}<4.4 \cdot 10^{-3}\right)$. El modelo de Page entregó el mejor ajuste, mostrando valores de $\mathrm{R}^{2}$ superiores a $99 \%$ y $\mathrm{X}^{2}$ menores que $1.1 \cdot 10^{-3}$.

El secado en lecho fluidizado pulsante produjo una disminución del contenido de sulforafano en el producto deshidratado. Las pérdidas de sulforafano se minimizan al utilizar baja temperatura del aire de secado y alta velocidad de giro de la placa rotatoria. El modelo de regresión predijo que las condiciones de secado que maximizan el contenido de sulforafano son temperatura del aire de secado igual a $29^{\circ} \mathrm{C}$ y velocidad de giro de la placa rotatoria igual a $108 \mathrm{rpm}$. Estas condiciones se verificaron experimentalmente, resultando en un contenido de sulforafano igual a $1.5 \pm 0.1 \mu \mathrm{mol} / \mathrm{g}$ base seca, lo que representa un incremento del $25 \%$ con respecto al brócoli fresco.

\section{NOTACIÓN}

\section{Símbolos}

A Temperatura del aire de secado, ${ }^{\circ} \mathrm{C}$

a Parámetro de ajuste adimensional del modelo de Henderson y Pabis

B Velocidad de giro de la placa rogatoria, rpm 
b Parámetro de ajuste adimensional del modelo logarítmico

c Parámetro de ajuste adimensional del modelo logarítmico

k Número de parámetros independientes en el modelo de regresión

$\mathrm{K}_{\mathrm{H}} \quad$ Parámetro de ajuste del modelo de Henderson y Pabis, min $^{-1}$

$\mathrm{K}_{\mathrm{I}} \quad$ Parámetro de ajuste del modelo logarítmico, $\mathrm{min}^{-1}$

$\mathrm{K}_{\mathrm{N}} \quad$ Parámetro de ajuste del modelo de Newton, $\mathrm{min}^{-1}$

$\mathrm{K}_{\mathrm{P}} \quad$ Parámetro de ajuste del modelo de Page, $\mathrm{min}^{-n}$

Mo Contenido de humedad inicial, $\mathrm{g} \mathrm{g}^{-1}$

$\mathrm{M}_{\mathrm{cr}} \quad$ Contenido de humedad crítico, $\mathrm{g} \mathrm{g}^{-1}$

MR Razón de humedad adimensional

$\mathrm{M}_{\mathrm{t}} \quad$ Contenido de humedad en función del tiempo, $\mathrm{g} \mathrm{g}^{-1}$

$\mathrm{R}^{2} \quad$ Coeficiente de determinación, \%

[S] Contenido de sulforafano, $\mu \mathrm{mol} \mathrm{g}^{-1}$

$\mathrm{T} \quad$ Corrida experimental

$\mathrm{t} \quad$ Tiempo de secado, min

$X \quad$ Niveles codificados de los factores experimentales

$\hat{Y} \quad$ Valor de la variable respuesta predicho por el modelo de regresión

\section{Abreviaturas}

b.s. Base seca

HPLC Cromatografía líquida de alta precisión

\section{Letras griegas}

$\beta \quad$ Coeficientes del modelo de regresión

$\mathrm{X}^{2} \quad$ Estadístico chi-cuadrado

\section{AGRADECIMIENTOS}

Los autores agradecen el apoyo financiero del Programa Fondecyt a través del Proyecto Regular $\mathrm{N}^{\circ}$ 1130384, Proyecto Basal USA 1555 y Vridei 01711MO_PUBLIC, y a la Universidad de Santiago de Chile.

\section{REFERENCIAS}

AOAC. Official Methods of Analysis. Association of Official Analytical Chemists, Washington, D.C., U.S.A., $15^{\text {th }}$ Ed., 1298 (1990)

Aghbashlo, M., M.H. Kianmehr, S. Khani y M. Ghasemi. Mathematical modelling of thin layer drying of carrot, doi: 10.1007/s11694-016-9432-y, International Agrophysics, 23, 313-317 (2009)

Ah-Hen, K., C.E. Zambra, J. Agüero, A. Vega-Gálvez y R. Lemus-Mondaca. Moisture Diffusivity Coefficient and Convective Drying Modelling of Murta (Ugni mollinae Turcz): Influence of Temperature and vacuum on Drying Kinetics, doi: 10.1007/s11947-011-0758-5, Food and Bioprocess Technology, 6(4), 919-930 (2013)

Babalis, S.J. y V.G. Belessiotis. Influence of the drying conditions on the drying constants and moisture diffusivity during the thin-layer drying of figs, doi: 10.1007/s13197-011-0399-8, Journal of Food Engineering, $65,449-458$ (2004)

Henderson, S.M. Progress in developing the thin layer drying equation, doi: 10.13031/2013.37052, Transactions of the ASAE, 17, 1167-1172 (1974)

Karathanos, V.T. Determination of water content of dried fruits by drying kinetics, doi: 10.1016/S02608774(98)00132-0, Journal of Food Engineering, 39, 337-344 (1999)

Lekcharoenkul, P., Y. Tanongkankit, N. Chiewchan y S. Devahastin. Enhancement of sulforaphane content in cabbage outer leaves using hybrid drying technique and stepwise change of drying temperature, doi: 10.1016/j.jfoodeng.2013.08.037, Journal of Food Engineering, 122, 56-61 (2014)

Lemus-Mondaca, R., N. Betoret, A. Vega-Galvéz y E. Lara-Aravena. Dehydration characteristics of papaya (carica pubescens): determination of equilibrium moisture content and diffusion coefficient, doi: 10.1111/j.1745-4530.2007.00236.x, Journal of Food Process Engineering, 32, 645-663 (2009)

Liang, H., Q. P. Yuan, H. R. Dong, y Y. M. Liu. Determination of sulforaphane in broccoli and cabbage by high-performance liquid chromatography, doi: 10.1016/j.jfca.2005.11.005, Journal of Food Composition and Analysis, 19 (5), 473-476 (2006) 
Mahn, A. y C. Pérez. Optimization of an incubation step to maximize sulforaphane content in pre-processed broccoli, doi: 10.1007/s13197-016-2386-6, J. Food Science and Technology, 53(11), 4110 - 4115 (2016)

Mahn, A., C. Martin, A. Reyes y A. Saavedra. Evolution of sulforaphane content in sulforaphane-enriched broccoli during tray drying, doi: 10.1016/j.jfoodeng.2016.04.007, Journal of Food Engineering, 186, 27-33 (2016a)

Mahn, A., J. Román y A. Reyes. Efecto de la Liofilización de Brócoli Pre-Procesado sobre la Cinética de Secado y el Contenido de Sulforafano, doi: 10.4067/S0718-07642016000600010, Información Tecnológica, 27(6), 95 - 106 (2016b)

Matusheski, N.V., J.A. Juvik y E.H. Jeffery. Heating decreases epithiospecifier protein activity and increases sulforaphane formation in broccoli, doi: 10.1016/j.phytochem.2004.04.013, Phytochemistry, 65 (9), 12731281 (2004)

Mazo, P.C., G.M. Restrepo, L.A. Ríos y J.M. Marín. Optimización de la Producción de Alquil Ésteres a partir de Aceite de palma, empleando la Metodología de Superficie de Respuesta, doi: 10.1612/inf.tecnol.3954it.07, Información Tecnológica, 19 (5), 37-48 (2008)

Mulet, A., N. Sanjuan, J. Bon y S. Simal. Drying model for highly porous hemispherical bodies, doi: 10.1007/s002170050538, European Food Research and Technology, 210, 80-83 (1999)

Page, G. E. Factors influencing the maximum rates for air drying shelled corn in thin layers. M.S. Thesis, Department of Mechanical Engineering, Purdue University, Purdue, U.S.A. (1949)

Pérez, C., H. Barrientos, J. Román, y A. Mahn. Optimization of a blanching step to maximize sulforaphane synthesis in broccoli florets, doi: 10.1016/j.foodchem.2013.08.053, Food Chemistry, 145, 264-271 (2014)

Reyes, A., A. Mahn, C. Guzmán y D. Antoniz. Analysis of the drying of broccoli florets in a fluidized pulsed bed, doi: 10.1080/07373937.2012.686548, Drying Technology, 30, 11-12 (2012)

Reyes, A., P. Moyano y J. Paz. Drying of Potato Slices in a Pulsed Fluidized Bed, doi: 10.1080/07373930701227011, Drying Technology, 25, 581-590 (2007)

Reyes, A., R. Vega, R. Bustos y C. Araneda. Effect of Processing Conditions on Drying Kinetics and Particle Microstructure of Carrot, Drying Technology, 26 (10), 1272-1285 (2008)

Senadeera, W., B.R. Bhandari., G. Young y B. Wijesinghe. Influence of shapes of selected vegetable material son drying kinetics during fluidized bed drying, doi: 10.1016/S0260-8774(02)00386-2, Journal of Food Engineering, 58 (3), 277-283 (2003)

Shibata, T., Y. Kimura, A. Mukai, H. Mori, S. Ito, Y. Asaka, S. Oe, H. Tanaka, T. Takahashi y K. Uchida. Transthiocarbamoylation of Proteins by Thiolated Isothiocyanates, doi: 10.1074/jbc.M111.308049, The Journal of Biological Chemistry, 286 (49), 42150-42161 (2011)

Tanongkankit, Y., N. Chiewchan, y S. Devahastin. Evolution of anticarcinogenic substance in dietary fiber powder from cabbage outer leaves during drying, doi: 10.1007/s13197-013-1203-8, Food Chemistry, 127 (1), 67-73 (2011)

Uribe, E., A. Vega-Gálvez, K. Di Scala, R. Oyanadel, J. S. Torrico y M. Miranda. Characteristics of Convective Drying of Pepino Fruit (Solanum muricatum Ait.): Application of Weibull Distribution, doi: 10.1007/s11947-009-0230-y, Food and Bioprocess Technology, 4, 1349-1356 (2011)

Vega, A.A. y R. A. Lemus. Modelado de la Cinética de Secado de la Papaya Chilena (Vasconcellea pubescens), doi: 10.4067/S0718-07642006000300005, Información Tecnológica, 17 (3), 23-31 (2006)

Vega-Gálvez, A., R. Lemus, C. Bilbao, F. Yagnam y A. Rojas. Mass transfer kinetics during convective drying of red pepper var. hungarian (capsicum annuum I.): mathematical modeling and evaluation of kinetic parameters, doi: 10.1111/j.1745-4530.2007.00145.x. J. Food Process Engineering, 31,120-137 (2008)

Wang, G.C., M. Farnham y E. H. Jeffery. Impact of Thermal Processing on sulforaphane Yield from Broccoli (Brassica oleracea L. ssp. italica), doi: 10.1021/jf2050284, Journal of Agricultural and Food Chemistry, 60 (27), 6743-6748 (2012)

Zambrano, M.L., D.B. Rodríguez y A. Álvarez. Estudio Cinético y de Superficie de Respuesta para la Rehidratación de Zanahorias (Daucus carota) Liofilizadas, doi: 10.4067/S071807642007000400008, Información Tecnológica, 18 (4), 47-56 (2007) 